\title{
COVID-19 relapse with prolonged viral shedding up to 60 days or re-infection, in 3 frontline healthcare workers with recurrent symptoms and persistent SARS-CoV-2 PCR positivity in Ireland, a developing diagnostic challenge: A case report
}

Jonathan McGrath ( $\nabla$ jonathan.mcgrath@umail.ucc.ie)

Mater Misericordiae University Hospital https://orcid.org/0000-0001-8227-0976

Tara McGinty

Mater Misericordiae University Hospital

Maureen Lynch

Mater Misericordiae University Hospital

Edel O'Regan

Mater Misericordiae University Hospital

Dominic Natin

Mater Misericordiae University Hospital

Peter O'Gorman

Mater Misericordiae University Hospital

John S Lambert

Mater Misericordiae University Hospital https://orcid.org/0000-0001-5404-2415

\section{Case Report}

Keywords: COVID-19, severe acute respiratory syndrome coronavirus 2, polymerase chain reaction, antibodies, health personnel, threshold cycle value, case report

Posted Date: June 11th, 2020

DOI: https://doi.org/10.21203/rs.3.rs-34532/v1

License: (9) This work is licensed under a Creative Commons Attribution 4.0 International License.

Read Full License 


\section{Abstract}

\section{Background:}

To date, the Corona Virus Disease-2019 (COVID-19) pandemic has resulted in more than 24,400 confirmed cases in Ireland, with more than $30 \%$ involving Healthcare Workers (HCW).

As more staff become involved in the care of COVID-19 patients, many key clinical considerations remain uncertain, including the possibility of re-infection following initial illness, the clinical significance of prolonged viral shedding and the degree of protection conferred by development of anti-Severe Acute Respiratory Syndrome Coronavirus 2 (SARS-CoV-2) antibodies.

We present 3 cases of COVID19-infected HCWs, each with distinct episodes of recurrent symptoms following initial resolution and with persistently positive SARS-CoV-2 PCR results, ranging up to 60 days post onset of illness. PCR results, cycle threshold (Ct) values and clinical assessment are provided to discuss the diagnostic difficulties in assessing relapsed COVID-19 infection, or re-infection with new virus following return to work.

\section{Case presentations:}

Patient 1,2 and 3 (age range 25-36) tested positive for SARS-CoV-2 via rtPCR on oro/nasopharyngeal swab with initial Ct values of 21.72, 24.52 and 26.58 respectively, following presentation with respiratory symptoms. All completed 14 day periods of self-isolation with full resolution of symptoms. Each patient has a clinical role and was involved in the management of COVID-19 patients following return to work. Patient 1 was admitted to hospital 44 days after initial illness, with cough, dyspnoea and a concurrent diagnosis of neurosyphilis. SARS-CoV-2 PCR was positive with Ct value 31.36 and remained positive for at least 60 days following initial illness onset. A full clinical recovery followed. Patients 2 and 3 represented to the Emergency Department with recurrent respiratory symptoms 29 and 40 days following initial illness onset respectively. SARS-CoV-2 PCR was demonstrated in each with Ct values 31.16 and 30.72 respectively. Each subsequently made a full recovery following a second period of self-isolation. Anti-SARS-CoV-2 IgG was demonstrated in all 3 patients.

\section{Conclusions:}

These cases demonstrate the diagnostic difficulties in determining intermittent presentation of COVID-19 infection with prolonged viral shedding, or re-infection with new virus following return to work. As the pandemic progresses, this represents a growing diagnostic challenge impacting patient assessment, staff deployment following illness and infection control.

\section{Background}

Up to May $23^{\text {rd }} 2020$, the Corona Virus Disease 19 (COVID-19) pandemic has resulted in more than 24,400 confirmed cases in Ireland, with 7,813 (32\%) involving Healthcare Workers (HCW)(1). Despite a 
growing evidence base, a number of key clinical considerations remain uncertain that are of particular relevance to frontline staff, regarding both clinical care and broader infection control concerns.

The possibility of re-infection with COVID-19 following initial illness remains in question as does the clinical significance of prolonged viral shedding. Development of antibodies against Severe Acute Respiratory Syndrome Coronavirus 2 (SARS-CoV-2) is well documented (2), but whether this form of response confers protective immunity, or over what timeframe has yet to be firmly established. As more staff become involved in the care of COVID-19 patients, the answers to these pertinent questions are of growing importance and urgency.

We present 3 cases of COVID19-infected healthcare workers with patient-facing roles, each with distinct episodes of recurrent symptoms, following initial resolution and with persistently positive SARS-CoV-2 PCR results, ranging up to 60 days post onset of illness.

\section{Case Presentations}

\section{Case 1}

In mid-March 2020, a 29 year old HCW presented to a community testing centre reporting a 7 day history of fever, dry cough, dyspnoea, sore throat and arthralgia. SARS-CoV-2 RNA was detected on oro/nasopharyngeal swab via reverse transcription polymerase chain reaction (rtPCR) with a cycle threshold (Ct) value of 21.72. The severity of symptoms and presence of normal vital signs meant that hospital admission was not indicated and the patient was placed in self-isolation for 14 days.

Past medical history included epilepsy, for which he took Sodium Valproate 500mg BD and asthma, for which he took Budesonide/Formoterol $160 \mathrm{mcg} / 4.5 \mathrm{mcg}$ BD and Salbutamol $100 \mathrm{mcg}$ PRN.

During isolation, the patient made a good clinical recovery with complete resolution of symptoms at least five days prior to completion.

On returning to work, 21 days after initial symptom onset, he was stationed on a dedicated COVID-19 ward providing care for infected patients. There were no patients under his care receiving non-invasive ventilation.

Two days later he experienced blurring of vision in his left eye, pain on eye movements and pre-orbital headache. Eight days following this, he noticed a recurrence, though mild, of dry cough and exertional dyspnoea. Ophthalmological assessment revealed bilateral uveitis. A repeat swab taken due to recurrent respiratory symptoms, detected SARS-CoV-2 with a Ct value of 31.36, 30 days after the initial result and 36 days after initial onset of symptoms. Prednisolone acetate $1 \%$ eye drops were prescribed as a topical agent for the uveitis and on the advice Infectious Diseases services, he entered a second 14-day selfisolation period, pending serology results. 
Eight days into isolation, he was admitted to hospital with an erythematous, macular rash affecting his chest/back, tinnitus, bilateral pre-orbital headache and vertigo. Ongoing dry cough and exertional dyspnoea was also reported. He was not in respiratory distress with RR $28 / \mathrm{min}, \mathrm{O}_{2} 97 \%$ on room air (RA), $\mathrm{BP} 117 / 65 \mathrm{mmHg}, \mathrm{HR} 87 \mathrm{bpm}$ and a temperature of $36.1^{\circ} \mathrm{C}$. Lung auscultation did not reveal any crepitations or wheeze. Cranial nerve exam revealed mild pain on lateral eye movements. Routine bloodwork was unremarkable and Chest X-Ray was clear.

Serology demonstrated a positive Rapid Plasma Reagin (RPR) 1:128, Syphilis IgM ElA and T. pallidum particle agglutination at 1:20480. HIV 1+2 antigen/antibody was negative. Serology results coupled with rash indicated secondary syphilis. Due to ocular involvement, a diagnosis of neurosyphilis was made, with a background of possible persistent or recurrent COVID-19 infection. Lumbar puncture revealed a clear CSF with 0 WCC/RCC, protein $348 \mathrm{mg} / \mathrm{L}$, glucose $3.2 \mathrm{mmol} / \mathrm{L}$ (serum glucose $6.2 \mathrm{mmol} / \mathrm{L}$ ). CSF VDRL and SARS-CoV-2 PCR were negative. The patient commenced treatment with intravenous Penicillin G.

Over the course of his 14-day inpatient stay, the patient remained positive for SARS-CoV-2 on repeated oro/nasopharyngeal swabs despite full resolution of respiratory symptoms, including one performed 53 days following his initial positive result and 60 days following his initial symptoms in March (Figure 1.). A final SARS-CoV-2 PCR carried out at day 66 yielded a negative result. A point of care rapid antibody test proved positive for anti-SARS-CoV-2 IgG, 59 days following onset of symptoms.

\section{Case 2}

In late March, a 36 year old HCW tested positive for COVID-19 with a positive SARS-CoV-2 RNA oro/nasopharyngeal swab, with a Ct value 24.52, after presenting with pyrexia, cough, dyspnoea and anosmia. The severity of her respiratory symptoms did not warrant hospital admission and as a result, she entered into a 14-day self-isolation period. Full subjective resolution of symptoms was reported at least 3 days prior to completion of her self-isolation. After a further 7 days ( 21 days from time of original swab) the patient returned to work.

This patient was a non-smoker with mild asthma for which she was prescribed Budesonide/Formoterol BD and Salbutamol 100mcg PRN.

In late April, 29 days after her initial positive result, the patient presented to the Emergency Department reporting acute onset of cough, dyspnoea, headache, anosmia and subjective chills/pyrexia (selfmeasured at $37.7^{\circ} \mathrm{C}$ ) ongoing for the preceding 24 hours. Vital signs showed $\mathrm{O}_{2}$ saturations $100 \%$ on RA, RR 30/min with difficulty completing normal sentences, HR 80bpm, BP 121/76 $\mathrm{mmHg}$ and temperature $36^{\circ} \mathrm{C}$. Lung auscultation revealed mild expiratory wheeze and decreased air entry bilaterally. Baseline laboratory findings were unremarkable with normal white cells/inflammatory markers and Chest X-Ray. An oro/nasopharyngeal swab taken on admission detected SARS-CoV-2 RNA, with a Ct value of 31.16. The patient was diagnosed with a mild-moderate asthma exacerbation and possible recurrent/unresolved 
COVID-19 infection. Standard asthma exacerbation treatment was prescribed, with nebulised bronchodilators and oral prednisolone. There was no indication for systemic antibiotics or directed COVID-19 therapy. A good clinical improvement was achieved and the patient was discharged 48 hours later to complete a second period of self-isolation with remote monitoring, via a smartphone application, measuring oxygen saturation/temperature twice daily as per local protocols. Dyspnoea resolved after a further 7 days (Figure 1). Anti-SARS-CoV-2, antibodies were detected using a point of care lateral flow test kit 51 days after initial symptom onset.

\section{Case 3}

In mid-March, a 25 year old HCW with no significant past medical history presented to the Emergency Department with fever, sore throat, dry cough, fatigue and myalgia on a background of recent travel to Italy. rtPCR testing for SARS-CoV-2 was positive, with a Ct value 26.58. Vital signs were all within normal range, laboratory investigations and Chest X-Ray were unremarkable. Following a 24-hour period of observation, the patient was discharged for a planned 14 days of self-isolation. The patient had an uneventful clinical course, reported full resolution of symptoms, except for ongoing fatigue, and returned to work 19 days after initial symptom onset.

In mid-April, 36 days following the initial positive swab result and 40 days following the initial onset of symptoms, the patient re-presented to the Emergency Department reporting a 5 day history of mild dry cough, myalgia and 10 days of sore throat/coryzal symptoms. Vital signs showed $\mathrm{O}_{2} 99 \%$ on RA, RR $16 / \mathrm{min}, \mathrm{HR} 122$, BP $156 / 98 \mathrm{~mm} / \mathrm{Hg}$ and temperature $37.1^{\circ} \mathrm{C}$. Chest X-Ray revealed a focus of patchy opacification in the medial right lower zone. Repeat SARS-CoV-2 PCR was positive, with a Ct value of 30.72. A nasopharyngeal aspirate reported not detected for a broad range of other respiratory viruses. The severity of symptoms did not warrant admission and the patient was discharged for a further 14 days self-isolation. Anti-SARS-CoV-2 IgG was detected via point of care testing 58 days post original positive swab result. The patient made a full clinical recovery (Figure 1).

\section{Discussion And Conclusions}

We describe here 3 cases of COVID-19 in frontline HCWs with distinct episodes of recurrent symptoms following a subjective resolution of their initial syndrome. It is not clear whether this represents a relapse of symptoms (intermittent presentation) of COVID-19 infection with prolonged viral shedding, or reinfection with new virus following return to work.

The reported symptom-free period between episodes was a minimum 15, 18, and 18 days, for Patient 1,2 and 3 respectively. All 3 patients worked in separate hospitals, with clinical, patient-facing roles and reported appropriate PPE use, without known breaches, for contact with known COVID-19 patients. Patient 3 was defined as a close contact of a known case in the intervening period between her two illnesses. 
Duration of SARS-CoV-2 PCR positivity varies, with one review reporting a median of 12 days (range 5 to 52 days) across a number of studies (3). Prolonged PCR positivity is demonstrated in our 3 cases, with detectable SARS-CoV-2 RNA at 53, 29 and 36 days post initial results for patient 1,2 and 3 respectively. The longest detectable period, in patient 1, was 60 days after the onset of his original symptoms. To the best of our knowledge, this represents one of the longest durations of SARS-CoV-2 PCR positivity reported (4).

All 3 patients were young (range 29 to 36 years) and had mild disease at initial presentation defined by symptom severity, normal vital signs and radiology (5). Patients 2 and 3 had a history of asthma and took regular inhaled corticosteroids, but no other significant co-morbidities were noted. While severe disease, ICU admission (6) and old age (7) have all been associated with prolonged viral shedding, a cohort study of 23 patients by Kai-Wang et al.(8) demonstrated viral RNA detection in saliva after 20 or more days in $23 \%$ of mild cases. One study (7) of 262 COVID-19 patients observed that those who tested positive following discharge tended to be younger, with milder forms of COVID-19 during their initial hospitalisation; however in this cohort, patients remained asymptomatic, while the three patients reported above had 2 distinct symptomatic presentations.

We analysed Ct values for each of our cases, where data was available, to explore the concept of prolonged viral shedding. Ct values for each successive result demonstrate a progressive increase for all patients. It is possible that this represents a reducing viral load from the original infection, but increasing Ct values alone do not however, out rule re-infection with the secondary episodes. Additionally, these results are not routinely available to clinicians at point of diagnosis and therefore cannot be used to aid clinical decisions at initial patient assessment.

A limitation in the interpretation of our findings is the lack of a negative SARS-CoV-2 PCR post initial symptom resolution in our 3 patients. However, a single negative PCR result needs careful interpretation; Xiao et al.(9), in a study of 70 patients, observed that $21.4 \%$ experienced a "turn positive" of nucleic acid detection by rtPCR for SARS-CoV-2 after two consecutive negative results. The author suggests this may be related to a false negative of the rtPCR test and prolonged nucleic acid conversion. While there is increasing published data documenting prolonged SARS-CoV-2 PCR detection, our cases demonstrate this phenomenon concurrently with recurrent symptoms, making clinical interpretation of its consequence more challenging.

Presence of anti-SARS-CoV-2 IgG was demonstrated in these patients via point of care, lateral flow SARSCoV-2 IgM/IgG Antibody Detection Kits. Due to the timing of patient presentation during the pandemic, quantitative measurement was not available during the acute episodes and as such, IgG presence was confirmed 59, 51 and 61 days after initial symptom onset in Patient 1, 2 and 3 respectively. Kai-Wang et al.(8) demonstrated that despite development of antibodies against SARS-CoV-2, viral RNA could still be detected in oropharyngeal saliva samples from a third of patients for 20 days or longer. Therefore, had antibody testing been available earlier in the clinical course for these patients, it is unlikely that it would have been able to fully clarify the relapse/re-infection issue, as antibodies may be positive even during 
productive COVID-19 infection. Similarly, Long et. Al (2) demonstrated that the proportion of patients with positive virus-specific IgG reached $100 \%$ approximately $17-19$ days after symptom onset, however, this did not necessarily equate to clinical recovery. Our 3 cases had mild severity illness and as such were able to return to work soon after their isolation was completed. It may be possible that potential reexposure to the virus occurred before adequate immune responses had developed and conferred protection. Wu et al.(10) found that while detectable, $30 \%$ of 175 studied patients failed to develop high levels of neutralising antibodies following recovery from COVID-19 infection, and levels were lower in those with less severe disease. Thus detection of antibodies, while promising, may not yet be a firm indicator of protection against re-infection with SARS-CoV-2.

A number of other limitations are noted in this patient analysis. First, the number of cases described is small and larger cohort studies are required to further clarify the issues raised. Furthermore, while antiSARS-CoV-2 IgG was demonstrated in all patients, direct temporal links to symptoms cannot be established due to lack of availability of antibody testing at time of presentation.

As the COVID-19 pandemic progresses, frontline workers will have increasing and repeated exposure to SARS-CoV-2 in occupational settings. These cases demonstrate that uncertainty remains regarding possibility of re-infection and clinical infectivity in prolonged shedding. This, coupled with the ongoing concern regarding neutralising antibody protection, means clinical assessment of this cohort may remain a challenge, impacting on diagnostics, isolation duration and re-deployment following illness.

\section{List Of Abbreviations}

COVID-19 - Coronavirus disease 2019

HCW - Healthcare worker

SARS-CoV-2 - Severe acute respiratory syndrome coronavirus 2

rtPCR- Reverse transcription polymerase chain reaction

Ct value - Cycle Threshold value

RPR: Rapid Plasma Reagin

CSF - Cerebrospinal fluid

VDRL - Venereal Disease Research Laboratory test

PPE - Personal protective equipment

\section{Declarations}

Ethics approval and consent to participate 
Not applicable

\section{Consent for publication}

All patients gave written informed consent for cases to be published

\section{Availability of data and materials}

Not applicable

\section{Competing interests}

No competing interests identified

\section{Funding}

Not applicable

\section{Authors' contributions}

JMcG analysed data and wrote manuscript

TMcG was a major contributor in writing manuscript

$M L$ identified and interpreted laboratory values, contributed to manuscript

EO'R identified and interpreted laboratory values

PO'G identified and interpreted laboratory values

DN identified and interpreted laboratory values

JSL concept formation, contributed to manuscript

All authors read and approved the final manuscript.

\section{Acknowledgements}

Not applicable

\section{References}

1. Health Protection Surveillance Centre (May 2020).Epidemiology of COVID-19 in Ireland. https://www.hpsc.ie/az/respiratory/coronavirus/novelcoronavirus

2. Long, Q., Liu, B., Deng, H. et al. Antibody responses to SARS-CoV-2 in patients with COVID-19. Nat Med (2020). https://doi.org/10.1038/s41591-020-0897-1 
3. Health Information and Quality Authority (April 2020). Evidence summary for COVID-19 viral load over course of infection. https://www.hiqa.ie/reports-and-publications/health-technologyassessment/evidence-summary-covid-19-viral-load-over

4. Li J, Zhang L, Liu B, Song D. Case Report: Viral Shedding for 60 Days in a Woman with Novel Coronavirus Disease (COVID-19) [published online ahead of print, 2020 Apr 27]. Am J Trop Med Hyg. 2020;10.4269/ajtmh.20-0275. doi:10.4269/ajtmh.20-0275

5. int. (2020). Clinical Management of Severe Acute Respiratory infection (SARI) When COVID-19 Disease is Suspected Interim Guidance 13 March 2020.https://www.who.int/docs/defaultsource/coronaviruse/clinical-management-of-novel-cov.pdf?sfvrsn=bc7da517_10\&download=true

6. Chen J, Qi T, Liu L, Ling Y, Qian Z, Li T, et al. Clinical progression of patients with COVID-19 in Shanghai, China. Journal of Infection. 2020

7. An J, Liao X, Xiao T, Qian S, Yuan J, Ye H, et al. Clinical characteristics of the recovered COVID-19 patients with re-detectable positive RNA test. medRxiv.2020:2020.03.26.20044222.

8. To KK-W, Tsang OT-Y, Leung W-S, Tam AR, Wu T-C, Lung DC, et al. Temporal profiles of viral load in posterior oropharyngeal saliva samples and serum antibody responses during infection by SARSCoV-2: an observational cohort study. The Lancet Infectious Diseases. 2020

9. Xiao, A.T., Tong, Y.X. and Zhang, S. (2020), False-negative of RT-PCR and prolonged nucleic acid conversion in COVID-19: Rather than recurrence. J Med Virol. Accepted Author Manuscript. doi:10.1002/jmv.25855

10. Fan Wu, Aojie Wang, Mei Liu, Qimin Wang, Jun Chen et al. Neutralizing antibody responses to SARSCoV-2 in a COVID-19 recovered patient cohort and their implications. medRxiv 2020.03.30.20047365; doi: https://doi.org/10.1101/2020.03.30.20047365

\section{Figures}




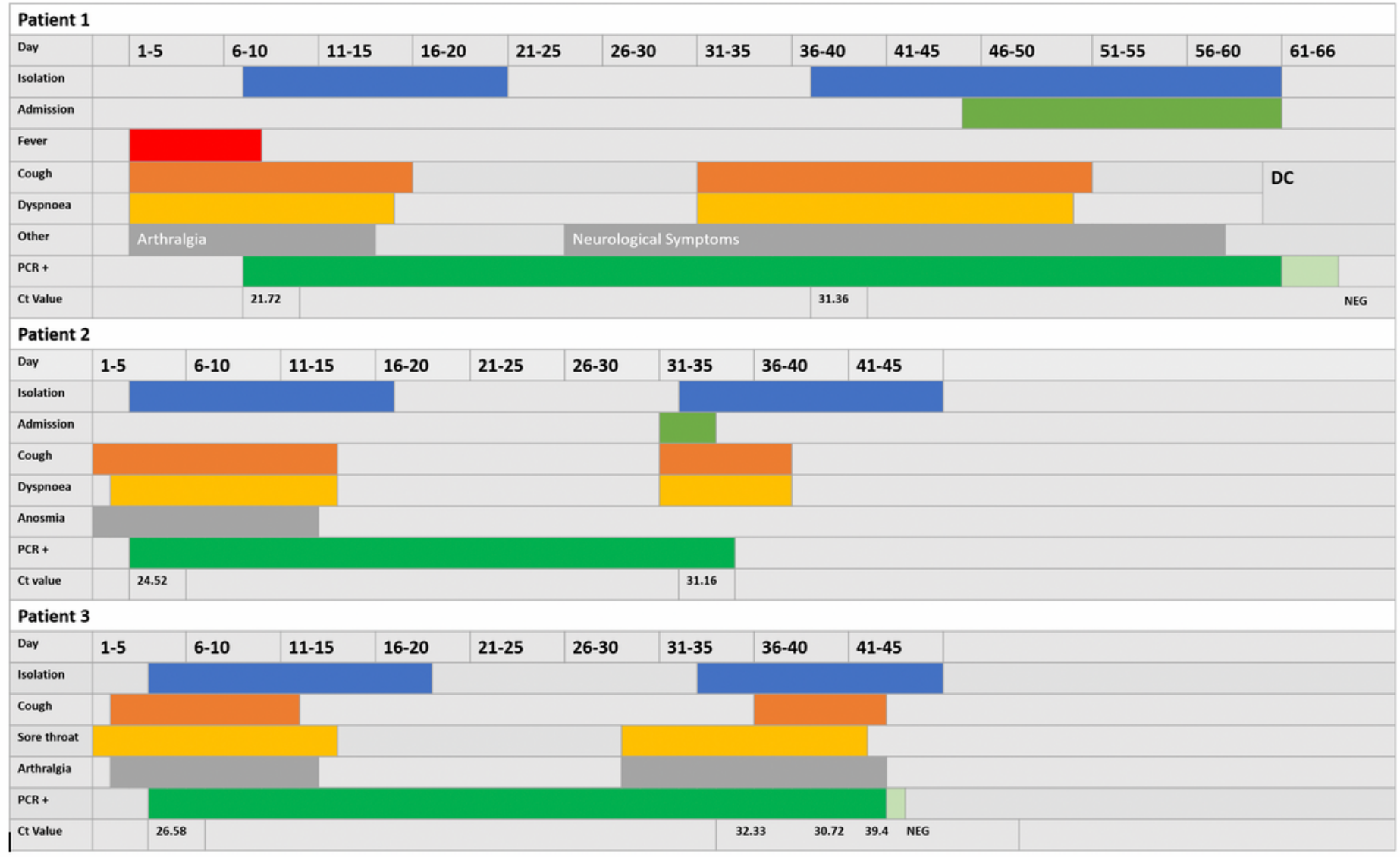

\section{Figure 1}

Timeline of predominant symptoms, isolation periods, hospital admission, SARS-CoV-2 polymerase chain reaction swab positivity $(\mathrm{PCR}+)$ and threshold cycle (Ct) values for Patient 1, 2 and 3 over the course of their illnesses. 